INPLASY

PROTOCOL

To cite: Ma et al. The efficacy and safety of Chinese patent medicines in the treatment of chronic atrophic gastritis: A bayesian network metaanalysis. Inplasy protocol 2020120063. doi:

10.37766/inplasy2020.12.0063

Received: 10 December 2020

Published: 11 December 2020

Corresponding author: Ma Yujing

1535392045@qq.com

Author Affiliation:

Hebei College of Traditional Chinese Medicine

Support: Natural Fund of Hebei Province.

Review Stage at time of this submission: Preliminary searches.

Conflicts of interest: None.

\section{The efficacy and safety of Chinese patent medicines in the treatment of chronic atrophic gastritis: A bayesian network meta-analysis}

\author{
Ma, J1; Liu, J2; Lang, X33 Kang, X4; Liu, X5; Jiao, $\mathrm{H}^{4}$.
}

Review question / Objective: P: Patients with chronic atrophic gastritis; I: Oral Chinese patent medicine combined with western medicine treatment; $\mathrm{C}$ : Oral Chinese patent medicine combined with western medicine or western medicine alone; 0 : Main outcome indicators: clinical efficacy.

Condition being studied: Chronic atrophic gastritis (CAG) is a type of chronic gastritis that is a chronic stomach disease in which repeated injury to the gastric mucosa epithelium results in a diminution of mucosal glands, or with intestinal metaplasia and/or pseudopyloric metaplasia.With the progression of the disease, CAG patients may have pathological changes such as thickening of mucosal muscle layer and intestinal metaplasia, increasing the risk of cancer.Western medicine has a single effect on CAG, and long-term use may cause adverse reactions, which makes the treatment difficult. In recent years, many clinical studies have shown that Chinese patent medicine combined with Western medicine has a significant effect on the treatment of CAG.

INPLASY registration number: This protocol was registered with the International Platform of Registered Systematic Review and Meta-Analysis Protocols (INPLASY) on 11 December 2020 and was last updated on 11 December 2020 (registration number INPLASY2020120063).

\section{INTRODUCTION}

Review question / Objective: P: Patients with chronic atrophic gastritis; I: Oral Chinese patent medicine combined with western medicine treatment; C: Oral Chinese patent medicine combined with western medicine or western medicine alone; 0: Main outcome indicators: clinical efficacy.

Condition being studied: Chronic atrophic gastritis (CAG) is a type of chronic gastritis that is a chronic stomach disease in which repeated injury to the gastric mucosa epithelium results in a diminution of 
mucosal glands, or with intestinal metaplasia and/or pseudopyloric metaplasia.With the progression of the disease, CAG patients may have pathological changes such as thickening of mucosal muscle layer and intestinal metaplasia, increasing the risk of cancer.Western medicine has a single effect on CAG, and long-term use may cause adverse reactions, which makes the treatment difficult. In recent years, many clinical studies have shown that Chinese patent medicine combined with Western medicine has a significant effect on the treatment of CAG.

\section{METHODS}

Participant or population: Patients with chronic atrophic gastritis.

Intervention: Oral Chinese patent medicine combined with western medicine treatment.

Comparator: Oral Chinese patent medicine combined with western medicine or western medicine alone.

\section{Study designs to be included: RCT.}

Eligibility criteria: Inclusion criteria Study type: Randomized Controlled Trial (RCT), regardless of whether blinding or allocation concealment is used. Participants: All patients diagnosed as CAG by endoscopic evaluation and mucosal biopsy. There are no restrictions on age 18 or older, gender, region, and other factors. Intervention The treatment group was treated with oral Chinese patent medicine combined with western medicine, and the control group was treated with oral Chinese patent medicine combined with western medicine or western medicine alone. The western medicine treatment group is consistent with the control group Exclusion criteria The research type is non-RCT, and nonclinical research types such as review, animal experiment and other chronic atrophic gastritis with severe complications cannot obtain the full text and repeated publication.
Information sources: Wanfang database, China National Knowledge Network (CNKI) database, China Biomedical Literature Service System (CBM) database, VIP Journal (VIP) database, PubMed database, Cochrane Library database, Embase database, International Clinical Trial Registry, China Clinical Trial Registry Center (from the establishment of the database until January 1, 2021), to retrieve randomized controlled trials of Chinese patent medicines in the treatment of chronic atrophic gastritis.

Main outcome(s): Main outcome indicators: clinical efficacy; secondary outcome indicators: gastroscopy efficacy, histopathological efficacy, TCM syndrome score, PGI, PGI/PGII, gastrin 17, Helicobacter pylori eradication rate, adverse reaction rate.

Quality assessment / Risk of bias analysis: Cochrane risk bias assessment tool.

Strategy of data synthesis: Stata $\mathbf{1 4 . 0}$ software was used to conduct a pairwise meta-analysis. The odds ratio (OR) was used as the effect indicator for binary categorical variables, and the weighted mean difference (MD) was used as the measurement indicator for continuous variables. Stata 14.0 software will be used, and random effects models will be introduced to merge data and draw evidence networks. We will perform the Bayesian network meta-analysis of the Markov chain Monte Carlo method (MCMC) in WinBUGS 1.4.3. The node split method will be used to evaluate the whole Consistency of a network.

Subgroup analysis: There are large differences between studies, analyze the source of heterogeneity, and conduct subgroup analysis.

Sensibility analysis: There are big differences between studies, analyze the source of heterogeneity, and conduct sensitivity analysis.

Country(ies) involved: China. 
Keywords: Chronic atrophic gastritis;

Chinese patent medicine; Bayesian network meta-analysis.

Contributions of each author:

Author 1 - Ma Yujing.

Email: 1535392045@163.com

Author 2 - Liu Jianping.

Email: 13603396653@163.com

Author 3 - Lang Xiaomeng.

Email: 171993143@qq.com

Author 4 - Kang Xin.

Email: kangxin0419@126.com

Author 5 - Liu Xioameng.

Email: 171993143@qq.com

Author 6 - Jiao Hong.

Email: 389545659@qq.com 